PROCEEDINGS OF THE

AMERICAN MATHEMATICAL SOCIETY

Volume 133, Number 4, Pages 1083-1091

S 0002-9939(04)07738-X

Article electronically published on November 19, 2004

\title{
POLYNOMIALS ON BANACH SPACES WITH UNCONDITIONAL BASES
}

\author{
BOGDAN C. GRECU AND RAYMOND A. RYAN
}

(Communicated by N. Tomczak-Jaegermann)

\begin{abstract}
We study the classes of homogeneous polynomials on a Banach space with unconditional Schauder basis that have unconditionally convergent monomial expansions relative to this basis. We extend some results of Matos, and we show that the homogeneous polynomials with unconditionally convergent expansions coincide with the polynomials that are regular with respect to the Banach lattices structure of the domain.
\end{abstract}

\section{INTRODUCTION}

Let $X$ be a Banach space with an unconditional Schauder basis. Every homogeneous polynomial or, equivalently, every multilinear form on $X$, has an expansion relative to this basis that converges pointwise with respect to a suitable ordering of the terms. It happens very rarely that all such expansions are unconditionally convergent, the space $\ell_{1}$ being a noteworthy exception. Closely related to this is the question of when the tensor product basis for a projective tensor power of $X$ is unconditional. Here, too, positive results are sparse [1] [4]. The existence of unconditionally convergent expansions is crucial in the study of holomorphic functions on spaces with unconditional Schauder bases, where one would seek to represent the function locally by monomial expansions. Motivated by these considerations, Matos [5, 6] isolated the classes of homogeneous polynomials and holomorphic functions that possess pointwise unconditionally convergent expansions, defined natural norms for these classes and uncovered some of their basic properties. In this paper we continue the study of these polynomials, and we show how his results can be understood in the wider context of Banach lattices.

\section{BILINEAR FORMS}

For the sake of clarity, we begin with the case of bilinear forms. Let $X, Y$ be Banach spaces over the real or complex numbers with 1-unconditional Schauder

Received by the editors November 19, 2003.

2000 Mathematics Subject Classification. Primary 46B15, 46G20; Secondary 46B42, 46B28.

Key words and phrases. Unconditional Schauder basis, homogeneous polynomial, tensor product.

The first author acknowledges the support of a Postdoctoral Fellowship funded by Enterprise Ireland.

The second author acknowledges the support of a Basic Research Grant from Enterprise Ireland.

(C)2004 American Mathematical Society

Reverts to public domain 28 years from publication 
bases $\left(e_{n}\right)$ and $\left(f_{n}\right)$ respectively. If $A$ is a bounded bilinear form on $X \times Y$, then $A$ is given pointwise by an iterated sum:

$$
A(x, y)=\sum_{n} \sum_{m} x_{n} y_{m} A\left(e_{n}, f_{m}\right),
$$

where $\sum_{n} x_{n} e_{n}$ and $\sum_{m} y_{m} f_{m}$ are the unconditionally convergent expansions of $x \in X$ and $y \in Y$. It is well known that this expansion need not be unconditionally convergent, even when $X, Y$ are Hilbert spaces and $\left(e_{n}\right),\left(f_{n}\right)$ are orthonormal bases [3].

We shall say that $A$ is an unconditional bilinear form if the above expansion of $A(x, y)$ converges unconditionally for every $(x, y) \in X \times Y$. The vector space of all unconditional bilinear forms will be denoted by $\mathcal{B}_{\nu}(X \times Y)$; of course, this space depends on the choice of bases, but our notation will be unambiguous since we always work with fixed bases. If $A$ is an unconditional form, then the bilinear form $B$ defined by

$$
B(x, y)=\sum_{n} \sum_{m}\left|A\left(e_{n}, f_{m}\right)\right| x_{n} y_{m}
$$

is also an unconditional form. Thus, a norm may be defined on the space $\mathcal{B}_{\nu}(X \times Y)$ by

$$
\nu(A)=\|B\|=\sup \left\{\sum_{n, m}\left|A\left(e_{n}, f_{m}\right)\right|\left|x_{n}\right|\left|y_{m}\right|:\|x\|,\|y\| \leq 1\right\} .
$$

This norm satisfies $\|A\| \leq \nu(A)$ and the space $\mathcal{B}_{\nu}(X \times Y)$ is complete in this norm. Furthermore, this space is never trivial; indeed, every nuclear bilinear form $A$ is unconditional and $\nu(A) \leq\|A\|_{N}$, the nuclear norm of $A$. We refer to [5, 6] for the proofs of these facts and the following examples.

If $X$ and $Y$ are $\ell_{1}$ with the standard basis, then every bounded bilinear form on $X \times Y$ is unconditional and the unconditional and uniform norms are equivalent. At the other extreme, if $X$ and $Y$ are $c_{0}$ with the standard basis, then the unconditional bilinear forms are precisely the nuclear forms and the unconditional and nuclear norms are equivalent.

For each bounded bilinear form $A$ on $X \times Y$, there is an associated operator $T: X \rightarrow Y^{*}$, given by

$$
\langle y, T x\rangle=A(x, y) .
$$

Nuclearity of the bilinear form $A$ is equivalent to nuclearity of the associated operator $T$. Thus, the result of Matos given above states that nuclearity of the associated operator is a sufficient condition for unconditionality. Our first proposition extends this idea.

Proposition 2.1. Let $X, Y$ be Banach spaces with 1-unconditional Schauder bases, and let $A$ be a bounded bilinear form on $X \times Y$. If the associated operator $T: X \rightarrow$ $Y^{*}$ is absolutely summing, then $A$ is unconditional.

Proof. Let $\left(e_{n}\right),\left(f_{n}\right)$ be 1-unconditional Schauder bases for $X, Y$ respectively. For every $x=\sum_{n} x_{n} e_{n} \in X$, the weak $\ell_{1}$-norm of the sequence $\left(x_{n} e_{n}\right)$ is given by

$$
\left\|\left(x_{n} e_{n}\right)\right\|_{1}^{w}=\sup \left\{\left\|\sum_{n} \lambda_{n} x_{n} e_{n}\right\|:\left|\lambda_{n}\right|=1\right\}=\|x\| .
$$


Hence, if $T$ is absolutely summing and $\left(y^{(n)}\right)$ is an arbitrary sequence in the closed unit ball of $Y$, then

$$
\sum_{n}\left|\left\langle y^{(n)}, T\left(x_{n} e_{n}\right)\right\rangle\right| \leq \sum_{n}\left\|T\left(x_{n} e_{n}\right)\right\| \leq \pi_{1}(T)\|x\| .
$$

Now let $x \in X, y \in Y$ and choose scalars $\varepsilon_{n m}$ of absolute value 1 so that $\left|A\left(e_{n}, f_{m}\right) x_{n} y_{m}\right|=\varepsilon_{n m} A\left(e_{n}, f_{m}\right) x_{n} y_{m}$. Then the vectors $y^{(n)}=\sum_{m} \varepsilon_{n m} y_{m} f_{m}$ all have the same norm as $y$ and so we have

$$
\begin{aligned}
\sum_{n, m}\left|A\left(e_{n}, f_{m}\right) x_{n} y_{m}\right| & =\sum_{n, m} \varepsilon_{n m} A\left(e_{n}, f_{m}\right) x_{n} y_{m}=\sum_{n} A\left(x_{n} e_{n}, y^{(n)}\right) \\
& =\sum_{n}\left\langle y^{(n)}, T\left(x_{n} e_{n}\right)\right\rangle \leq \pi_{1}(T)\|x\|\|y\| .
\end{aligned}
$$

Therefore $A$ is unconditional with $\nu(A) \leq \pi_{1}(T)$.

A bounded bilinear form is integral if and only if the associated operator is an integral operator. Since every integral operator is absolutely summing, we have the following special case of the proposition.

Corollary 2.2. Every integral bilinear form is unconditional.

The canonical injection of $\ell_{1}$ into $\ell_{2}$ is a well-known example of an absolutely summing operator that is not integral. Thus the bilinear form $A(x, y)=\sum_{n} x_{n} y_{n}$ on $\ell_{1} \times \ell_{2}$ (with the standard bases) is an example of an unconditional form that is not integral. We note that the sufficient condition given in the proposition is far from being necessary; every bounded bilinear form on $\ell_{1} \times \ell_{1}$ is unconditional, but there are many operators from $\ell_{1}$ into $\ell_{\infty}$ that are not absolutely summing.

Our next step is to show that the unconditional bilinear forms on $X \times Y$ are the bounded linear functionals on the tensor product $X \otimes Y$ with an appropriate norm. We recall that we are working with fixed 1-unconditional Schauder bases $\left(e_{n}\right),\left(f_{n}\right)$ for $X, Y$ respectively. Every $u \in X \otimes Y$ may be expressed uniquely as an iterated sum

$$
u=\sum_{n} \sum_{m} u_{n m} e_{n} \otimes f_{m}
$$

the coefficients being given by $u_{n m}=\left\langle u, e_{n}^{*} \otimes f_{m}^{*}\right\rangle$. We define

$$
\mu(u)=\sup \left\{\sum_{n, m}\left|u_{n m} A\left(e_{n}, f_{m}\right)\right|: A \in \mathcal{B}_{\nu}(X \times Y), \nu(A) \leq 1\right\} .
$$

To see that this quantity is finite, let $\sum_{j=1}^{k} x^{(j)} \otimes y^{(j)}$ be a representation of the tensor $u$. Then $u_{n m}=\sum_{j=1}^{k} x_{n}^{(j)} y_{m}^{(j)}$ and so we have

$$
\sum_{n, m}\left|u_{n m} A\left(e_{n}, f_{m}\right)\right| \leq \sum_{j} \sum_{n, m}\left|A\left(e_{n}, f_{m}\right)\right|\left|x_{n}^{(j)}\right|\left|y_{m}^{(j)}\right| \leq \sum_{j} \nu(A)\left\|x^{(j)}\right\|\left\|y^{(j)}\right\|,
$$

and it follows that $\mu(u) \leq \pi(u)$, where $\pi$ is the projective tensor norm.

Proposition 2.3. Let $X, Y$ be Banach spaces with 1-unconditional Schauder bases $\left(e_{n}\right),\left(f_{n}\right)$ respectively.

(a) $\mu$ is a reasonable crossnorm on $X \otimes Y$.

(b) The dual space of $X \otimes_{\mu} Y$ is isometrically isomorphic to the space $\mathcal{B}_{\nu}(X \times Y)$ of unconditional bilinear forms with the unconditional norm. 
Proof. It is easy to see that $\mu$ is a seminorm on $X \otimes Y$, and we have shown that $\mu \leq \pi$. For every $\varphi \in X^{*}, \psi \in Y^{*}$, the bilinear form $\varphi \otimes \psi$ has unconditional norm equal to $\|\varphi\|\|\psi\|$. It follows immediately that $\varepsilon \leq \mu$, where $\varepsilon$ is the injective norm. Therefore $\mu$ is a norm and $\varepsilon \leq \mu \leq \pi$ implies that $\mu$ is a reasonable crossnorm on $X \otimes Y$.

To prove the second assertion, let $A$ be an unconditional bilinear form on $X \times Y$. For every $u \in X \otimes Y$,

$$
|\langle u, A\rangle| \leq \sum_{n, m}\left|u_{n m}\right|\left|A\left(e_{n}, f_{m}\right)\right| \leq \nu(A) \mu(u)
$$

by the definition of $\mu(u)$, and so $A$ defines a bounded linear functional on $X \otimes_{\mu} Y$ with $\mu^{*}(A) \leq \nu(A)$.

Conversely, suppose that the bilinear form $A$ is bounded for the norm $\mu$ on $X \otimes Y$. Let $x \in X, y \in Y$. Then

$$
\begin{aligned}
\sum_{n, m}\left|A\left(e_{n}, f_{m}\right) x_{n} y_{m}\right| & \leq \mu\left(\sum_{n} \sum_{m} x_{n} y_{m} e_{n} \otimes f_{m}\right) \mu^{*}(A) \\
& =\mu(x \otimes y) \mu^{*}(A)=\|x\|\|y\| \mu^{*}(A) .
\end{aligned}
$$

Therefore $A$ is an unconditional form and $\nu(A) \leq \mu^{*}(A)$.

Together, these facts show that $\left(X \otimes_{\mu} Y\right)^{*}=\mathcal{B}_{\nu}(X \times Y)$ and the dual norm $\mu^{*}$ coincides with the unconditional norm $\nu$.

If $\alpha$ is a tensor norm, it happens very rarely that the tensors $e_{n} \otimes f_{m}$ form an unconditional basis for the Banach space $X \hat{\otimes}_{\alpha} Y$ [4]. However, the norm $\mu$ is not a tensor norm, since it depends on the choice of unconditional bases. For this norm, we have a very satisfactory result:

Corollary 2.4. The tensors $e_{n} \otimes f_{m}$ form a 1-unconditional Schauder basis for the Banach space $X \hat{\otimes}_{\mu} Y$.

Proof. Since the tensors $e_{n} \otimes f_{m}$ span a dense subspace of $X \hat{\otimes}_{\mu} Y$, it only remains to show that they are a 1-unconditional basic sequence. Let $I, J$ be finite subsets of the indexing set $\mathbb{N}^{2}$ with $I \subset J$, let $u_{n m}$ be scalars and let $\varepsilon_{n m}$ be scalars of absolute value 1. It follows from the definition of the norm $\mu$ that

$$
\mu\left(\sum_{(n, m) \in I} \varepsilon_{n m} u_{n m} e_{n} \otimes f_{m}\right) \leq \mu\left(\sum_{(n, m) \in J} u_{n m} e_{n} \otimes f_{m}\right),
$$

and we are done.

Let us denote by $\mathcal{B}_{\nu_{0}}(X \times Y)$ the closed subspace of $\mathcal{B}_{\nu}(X \times Y)$ spanned by the coordinate functionals $e_{n}^{*} \otimes f_{m}^{*}$. It follows from the above result that $\left(e_{n}^{*} \otimes f_{m}^{*}\right)$ is a 1-unconditional basis for the space $\mathcal{B}_{\nu_{0}}(X \times Y)$. We have a simple description of this space:

Proposition 2.5. A bilinear form $A$ belongs to the space $\mathcal{B}_{\nu_{0}}(X \times Y)$ if and only if the expansion $\sum_{n, m} A\left(e_{n}, f_{m}\right) x_{n} y_{m}$ is uniformly absolutely convergent on bounded subsets of $X \times Y$.

Proof. If $A$ belongs to $\mathcal{B}_{\nu_{0}}(X \times Y)$, then its expansion converges in the unconditional norm. Since this norm dominates the supremum norm, it follows that the expansion converges to $A$ uniformly on the product of the unit balls of $X$, 
$Y$ and hence uniformly on every bounded set. Conversely, suppose that the expansion $\sum_{n, m} A\left(e_{n}, f_{m}\right) x_{n} y_{m}$ is uniformly absolutely convergent on bounded sets. Then, given $\varepsilon>0$, there is a finite subset $J$ of $\mathbb{N} \times \mathbb{N}$ such that, if $I$ is any finite set of indices that is disjoint from $J$, then $\sum_{I}\left|A\left(e_{n}, f_{m}\right) x_{n} y_{m}\right|<\varepsilon$ for every $(x, y) \in B_{X} \times B_{Y}$. It follows from the definition of the unconditional norm that $\nu\left(\sum_{I} A\left(e_{n}, f_{m}\right) x_{n} y_{m}\right) \leq \varepsilon$, and we are done.

\section{Homogeneous Polynomials}

The results of the previous section extend easily to multilinear forms of arbitrary degree. For the sake of simplicity, let us consider $n$-linear forms on $X^{n}$, where $X$ has a fixed 1-unconditional Schauder basis $\left(e_{n}\right)$. Denoting by $\mathcal{L}\left({ }^{n} X\right)$ the Banach space of bounded $n$-linear forms on $X^{n}$, every $A \in \mathcal{L}\left({ }^{n} X\right)$ has an expansion

$$
A\left(x_{1}, \ldots, x_{n}\right)=\sum_{\left(j_{1}, \ldots, j_{n}\right) \in \mathbb{N}^{n}} A\left(e_{j_{1}}, \ldots, e_{j_{n}}\right) x_{1 j_{1}} \ldots x_{n j_{n}}
$$

that converges at each point when taken as an iterated sum. As for bilinear forms, we say that $A$ is unconditional if this expansion is unconditionally convergent at every point of $X^{n}$. The space $\mathcal{L}_{\nu}\left({ }^{n} X\right)$ of unconditional $n$-linear forms is a Banach space with the unconditional norm, defined as $\nu(A)=\|B\|$, where $B$ is the $n$-linear form with coefficients $B\left(e_{j_{1}}, \ldots, e_{j_{n}}\right)=\left|A\left(e_{j_{1}}, \ldots, e_{j_{n}}\right)\right|$.

A function $P$ on $X$ is an $n$-homogeneous polynomial if there exists a necessarily unique symmetric $n$-linear form $A$ such that $P(x)=A(x, \ldots, x)$ for every $x \in X$. We refer to $A$ as the symmetric generator of $P$. The $n$-homogeneous polynomial $P$ is bounded on the unit ball of $X$ if and only if $A$ is bounded. Furthermore, the supremum norm of $P$ satisfies

$$
\|P\| \leq\|A\| \leq \frac{n^{n}}{n !}\|P\| .
$$

We denote by $\mathcal{P}\left({ }^{n} X\right)$ the Banach space of $n$-homogeneous polynomials with this norm, and we refer to [2] for further facts about homogeneous polynomials. We have a coordinate expansion for any $n$-homogeneous polynomial $P$ that converges pointwise as an iterated sum:

$$
P(x)=A(x, \ldots, x)=\sum_{\left(j_{1}, \ldots, j_{n}\right) \in \mathbb{N}^{n}} A\left(e_{j_{1}}, \ldots, e_{j_{n}}\right) x_{j_{1}} \ldots x_{j_{n}}
$$

and we say that $P$ is unconditional if the convergence is unconditional at each point. The symmetry of $A$ implies that the coefficients in this expansion are symmetric in the sense that

$$
A\left(e_{j_{1}}, \ldots, e_{j_{n}}\right)=A\left(e_{k_{1}}, \ldots, e_{k_{n}}\right)
$$

whenever there is a permutation carrying the $n$-tuple $\left(j_{1}, \ldots, j_{n}\right)$ into the $n$-tuple $\left(k_{1}, \ldots, k_{n}\right)$. Collecting these coefficients together allows us to represent an unconditional polynomial with a monomial expansion:

$$
P(x)=\sum_{\alpha \in \mathbb{N}_{n}} c_{\alpha} x^{\alpha}
$$

where $\mathbb{N}_{n}$ denotes the set of multiindices $\alpha$ of degree $n$ and $x^{\alpha}$ is the product $\prod x_{j}^{\alpha_{j}}$, with the customary convention that $0^{0}=1$. The coefficients $c_{\alpha}$ that appear in this 
expansion are given by

$$
c_{\alpha}=\frac{n !}{\alpha_{1} ! \alpha_{2} ! \ldots} A\left(e_{1}^{\alpha_{1}} e_{2}^{\alpha_{2}} \ldots\right) .
$$

Conversely, if the monomial expansion of $P$ is pointwise unconditionally convergent, then it is easy to see that $P$ is an unconditional polynomial.

It follows that, if $P$ is unconditional, then an $n$-homogeneous polynomial $Q$ can be defined by

$$
Q(x)=\sum_{\alpha \in \mathbb{N}_{n}}\left|c_{\alpha}\right| x^{\alpha}=\sum_{\left(j_{1}, \ldots, j_{n}\right) \in \mathbb{N}^{n}}\left|A\left(e_{j_{1}}, \ldots, e_{j_{n}}\right)\right| x_{j_{1}} \ldots x_{j_{n}},
$$

and the unconditional norm of $P$ is then defined as $\nu(P)=\|Q\|$. Matos introduced this norm and showed that the space $\mathcal{P}_{\nu}\left({ }^{n} X\right)$ of unconditional $n$-homogeneous polynomials is complete in this norm [5, 6].

If $P$ is an $n$-homogeneous polynomial with symmetric generator $A$, then the associated operator $T_{P}: X \rightarrow \mathcal{P}\left({ }^{n-1} X\right)$ is defined by $T_{P}(x)(y)=A(x, y, \ldots, y)$. The following sufficient condition for unconditionality is the multilinear analogue of Proposition 2.1. The proof is essentially the same.

Proposition 3.1. Let $X$ be a Banach space with a 1-unconditional Schauder basis, and let $P$ be a bounded $n$-homogeneous polynomial on $X$. Then $P$ is unconditional if the associated operator $T_{P}$ is an absolutely summing operator from $X$ into $\mathcal{P}_{\nu}\left({ }^{n-1} X\right)$.

We have a useful special case:

Corollary 3.2. Every integral polynomial is unconditional.

Arguing as in the previous section, we define the subspace $\mathcal{P}_{\nu_{0}}\left({ }^{n} X\right)$ to be the closed subspace of $\mathcal{P}_{\nu}\left({ }^{n} X\right)$ spanned by the monomials $x^{\alpha}, \alpha \in \mathbb{N}_{n}$. Then the monomials are an unconditional Schauder basis for this space. When $X$ is $c_{0}$, only the nuclear polynomials are unconditional [5, 6], and so we have $\mathcal{P}_{\nu_{0}}\left({ }^{n} c_{0}\right)=$ $\mathcal{P}_{\nu}\left({ }^{n} c_{0}\right)=\mathcal{P}_{N}\left({ }^{n} c_{0}\right)$, with equivalence of norms. At the other extreme, we have observed that every bounded $n$-homogeneous polynomial on $\ell_{1}$ is unconditional. In this case, $\mathcal{P}_{\nu_{0}}\left({ }^{n} \ell_{1}\right)$ is the space of approximable polynomials, or, since $\ell_{\infty}$ has the approximation property, the space $\mathcal{P}_{w}\left({ }^{n} \ell_{1}\right)$ of $n$-homogeneous polynomials that are weakly continuous on bounded sets [2, p. 90].

\section{Polynomials on Banach lattices}

In this section, we show how the results of the previous sections can be understood in the wider context of Banach lattices. Every Banach space with a 1-unconditional Schauder basis is a Banach lattice, the lattice operations being defined coordinatewise. We work with lattices over the real field. We refer to [7, 9] for details of the results on Banach lattices that we use.

We recall that an operator $T: X \rightarrow Y$ between Banach lattices is positive if $T x \in Y_{+}$for every $x \in X_{+}$, where $X_{+}$denotes the positive cone of $X$. The operator $T$ is regular if it can be expressed as the difference of two positive operators. If $Y$ is Dedekind complete, then regularity of $T$ is equivalent to $T$ being order bounded; in other words, $T$ maps order bounded subsets of $X$ into order bounded subsets of $Y$. If $Y$ is Dedekind complete, then the regular operators from $X$ into $Y$ are bounded and form a Dedekind complete Banach lattice, $\mathcal{L}_{r}(X, Y)$, with norm the so-called 
regular norm, given by $\|T\|_{r}=\||T|\|$, where $|T|$ is the absolute value of $T$ with respect to the Banach lattice structure of $\mathcal{L}_{r}(X, Y)$. In particular, the Banach dual space of a Banach lattice $X$ coincides with the Dedekind complete Banach lattice of order bounded linear functionals on $X$.

We now consider multilinear forms on a Banach lattice $X$. An $n$-linear form $A$ on $X^{n}$ is positive if $A\left(x_{1}, \ldots, x_{n}\right) \geq 0$ whenever $x_{1}, \ldots, x_{n}$ lie in the positive cone of $X$. A partial order is defined on the space of $n$-linear forms by $A_{1} \geq A_{2}$ if $A_{1}-A_{2}$ is positive. An $n$-linear form is regular if it can be written as the difference of two positive forms.

If $A$ is an $n$-linear form on $X^{n}$, the associated linear mapping $A_{1}: X \rightarrow \mathcal{L}\left({ }^{n-1} X\right)$ is defined by

$$
A_{1}(x)\left(x_{1}, \ldots, x_{n-1}\right)=A\left(x, x_{1}, \ldots, x_{n-1}\right) .
$$

Proposition 4.1. Let $X$ be a real Banach lattice.

(a) Every regular $n$-linear form on $X^{n}$ is bounded.

(b) The set $\mathcal{L}_{r}\left({ }^{n} X\right)$ of regular $n$-linear forms on $X$ is a vector lattice.

(c) $\mathcal{L}_{r}\left({ }^{n} X\right)$ is a Dedekind complete Banach lattice with the norm $\|A\|_{r}=\||A|\|$, where $|A|$ is the absolute value of $A$ with respect to the vector lattice structure of $\mathcal{L}_{r}\left({ }^{n} X\right)$.

(d) An n-linear form $A$ on $X^{n}$ is regular if and only if the associated linear mapping $A_{1}$ is a regular operator from $X$ into $\mathcal{L}_{r}\left({ }^{n-1} X\right)$.

Proof. (a) It suffices to establish the result for positive forms. The proof is a straightforward modification of the corresponding fact for positive operators [7] p. 27].

(b) The proof is by induction on $n$. The case $n=1$ is clear, since $\mathcal{L}_{r}\left({ }^{1} X\right)$ is just the order dual of $X$. We complete the induction by showing that $\mathcal{L}_{r}\left({ }^{n} X\right)$ can be identified with the Riesz space $\mathcal{L}_{r}\left(X, \mathcal{L}_{r}\left({ }^{n-1} X\right)\right)$, which by the induction assumption is Dedekind complete. This identification is obtained by associating with each regular $n$-linear form $A$ the linear mapping $A_{1}: X \rightarrow \mathcal{L}\left({ }^{n-1} X\right)$. Let $A$ be regular, so that it can be expressed as $B-C$, where $B$ and $C$ are positive forms. Then $A_{1}=B_{1}-C_{1}$ and $B_{1}(x), C_{1}(x)$ are regular $(n-1)$-forms for every $x \in X$, since, for example, we may write $B_{1}(x)=B_{1}\left(x^{+}\right)-B_{1}\left(x^{-}\right)$, the difference of two positive forms. Therefore $A_{1}$ maps $X$ into $\mathcal{L}_{r}\left({ }^{n-1} X\right)$. Furthermore, $B_{1}$ and $C_{1}$ are positive operators and so $A_{1}$ is regular. Conversely, if $T$ is a regular operator from $X$ into $\mathcal{L}_{r}\left({ }^{n-1} X\right)$, then it is easy to see that the formula $A\left(x_{1}, x_{2}, \ldots, x_{n}\right)=$ $T\left(x_{1}\right)\left(x_{2}, \ldots, x_{n}\right)$ defines a regular $n$-linear form $A$ with $A_{1}=T$. Therefore $\mathcal{L}_{r}\left({ }^{n} X\right)$ and $\mathcal{L}_{r}\left(X, \mathcal{L}_{r}\left({ }^{n-1} X\right)\right)$ are isomorphic as vector spaces. Finally, the order relation defined by the positive cone consisting of the positive $n$-linear forms on $X^{n}$ coincides with the canonical ordering on the Riesz space $\mathcal{L}_{r}\left(X, \mathcal{L}_{r}\left({ }^{n-1} X\right)\right)$.

(c) and (d) follow from the above.

Now suppose that $X$ is a Banach space with a 1-unconditional Schauder basis $\left(e_{n}\right)$. If $A$ is a bounded $n$-linear form on $X^{n}$, then the coefficients in the coordinate expansion of $A$ are given by the values of $A$ on $n$-tuples of the form $\left(e_{j_{1}}, \ldots, e_{j_{n}}\right)$. Since the basis vectors lie in the positive cone of $X$, it follows that $A$ is positive if and only if every coefficient in the expansion of $A$ is nonnegative. Now it is easy to see that every positive form is unconditional; hence every regular form is unconditional. To establish the converse, suppose that $A$ is an unconditional 
$n$-linear form. Let

$$
A\left(x_{1}, \ldots, x_{n}\right)=\sum_{\left(j_{1}, \ldots, j_{n}\right) \in \mathbb{N}^{n}} a_{j_{1} \ldots j_{n}} x_{1 j_{1}} \ldots x_{n j_{n}}
$$

be the pointwise unconditionally convergent expansion of $A$. We define $n$-linear forms $A^{\prime}$ and $A^{\prime \prime}$ by

$$
A^{\prime}\left(x_{1}, \ldots, x_{n}\right)=\sum_{\left(j_{1}, \ldots, j_{n}\right) \in \mathbb{N}^{n}} a_{j_{1} \ldots j_{n}}^{+} x_{1 j_{1}} \ldots x_{n j_{n}}
$$

and

$$
A^{\prime \prime}\left(x_{1}, \ldots, x_{n}\right)=\sum_{\left(j_{1}, \ldots, j_{n}\right) \in \mathbb{N}^{n}} a_{j_{1} \ldots j_{n}}^{-} x_{1 j_{1}} \ldots x_{n j_{n}} .
$$

It follows from the unconditional convergence of the expansion of $A$ that these expansions converge unconditionally and define unconditional $n$-linear forms on $X^{n}$. Since $A^{\prime}, A^{\prime \prime}$ are positive and $A=A^{\prime}-A^{\prime \prime}$, our claim is proved. It is not difficult to show that the forms $A^{\prime}$ and $A^{\prime \prime}$ defined above are the positive and negative parts of $A$, and hence the absolute value of $A$ is given by

$$
|A|\left(x_{1}, \ldots, x_{n}\right)\left|=\sum_{\left(j_{1}, \ldots, j_{n}\right) \in \mathbb{N}^{n}}\right| a_{j_{1}} \ldots a_{j_{n}} \mid x_{1 j_{1}} \ldots x_{n j_{n}} .
$$

It follows that $\nu(A)=\|A\|_{r}$. Summarizing, we have

Proposition 4.2. Let $X$ be a real Banach space with a 1-unconditional Schauder basis. A bounded n-linear form on $X^{n}$ is unconditional if and only if it is regular. Furthermore, the unconditional and regular norms coincide.

We wish to transfer these results to $n$-homogeneous polynomials. Let $X$ be a real Banach lattice, and let $P$ be an $n$-homogeneous polynomial on $X$, generated by the symmetric $n$-linear form $A$. We shall say that $P$ is a positive polynomial if the corresponding symmetric multilinear form $A$ is positive. Note that this is stronger than requiring $P$ to take nonnegative values on the positive cone of $X$.

As for multilinear forms, a bounded $n$-homogeneous polynomial is said to be regular if it is the difference of two positive $n$-homogeneous polynomials. In the light of the above proposition, it is clear that the space of regular $n$-homogeneous polynomials, which we denote by $\mathcal{P}_{r}\left({ }^{n} X\right)$, is isomorphic as a vector lattice to the space of regular symmetric $n$-linear forms. Furthermore, $\mathcal{P}_{r}\left({ }^{n} X\right)$ is a Banach lattice for the regular norm:

$$
\|P\|_{r}=\||P|\|,
$$

where $|P|$ denotes the absolute value of $P$ with respect to the vector lattice structure we have defined.

Now suppose that $X$ has a 1-unconditional Schauder basis. Let $\sum c_{\alpha} x^{\alpha}$ be the monomial expansion of the $n$-homogeneous polynomial $P$, whose symmetric generator is $A$. It follows from our previous results that $P$ is an unconditional polynomial if and only if $P$ is regular. Furthermore, if $P$ is regular, then $|P|$ is given by the expansion

$$
|P|(x)=\sum_{\alpha}\left|c_{\alpha}\right| x^{\alpha}
$$


We summarize our findings:

Proposition 4.3. Let $X$ be a real Banach space with a 1-unconditional Schauder basis. Then the spaces of unconditional and regular $n$-homogeneous polynomials on $X$ are the same for every $n$ and the unconditional norm on $\mathcal{P}_{\nu}\left({ }^{n} X\right)=\mathcal{P}_{r}\left({ }^{n} X\right)$ is equal to the regular norm.

\section{REFERENCES}

1. A. Defant, J. C. Díaz, D. Garcia, M. Maestre, Unconditional Basis and Gordon-Lewis Constants for Spaces of Polynomials, J. Funct. Anal. 181 (2001), 119-145. MR1818112 (2002b:46030)

2. S. Dineen, Complex analysis on infinite-dimensional spaces, Springer Monographs in Mathematics, Springer-Verlag, London, 1999. MR1705327 (2001a:46043)

3. B. R. Gelbaum and J. Gil de Lamadrid, Bases of tensor products of Banach spaces, Pacific J. Math. 11 (1961), 1281-1286. MR0147881 (26:5394)

4. S. Kwapień and A. Pełczyński, The main triangle projection in matrix spaces and its applications, Studia Math. 34 (1970), 43-68. MR0270118 (42:5011)

5. M. Matos, On holomorphy in Banach spaces and absolute convergence of Fourier series, Portugal Math. 45 (1988), no. 4, 429-450. (See also Errata: "On holomorphy in Banach spaces and absolute convergence of Fourier series" Port. Math. 47 (1990), no. 1, 13.) MR0982911 (90f:46075); MR 1079501 (91j:46054)

6. M. Matos and L. Nachbin, Reinhardt domains of holomorphy in Banach spaces, Adv. Math. 92 (1992), no. 2, 266-278. MR.1155467 (93d:46069)

7. P. Meyer-Nieberg, Banach Lattices, Springer Verlag, Berlin, 1991. MR1128093 (93f:46025)

8. R. A. Ryan, Introduction to Tensor Products of Banach Spaces, Springer Verlag, 2002. MR1888309 (2003f:46030)

9. H. H. Schaefer, Banach Lattices and Positive Operators, Springer Verlag, New YorkHeidelberg, 1974. MR0423039(54:11023)

Department of Mathematics, National University of Ireland, Galway, Ireland

E-mail address: bogdan@wuzwuz.nuigalway.ie

Department of Mathematics, National University of Ireland, Galway, Ireland

E-mail address: ray.ryan@nuigalway.ie 